\title{
Lynch syndrome type II associated endometrial carcinoma: a case report and literature analysis
}

\author{
hui juan $\mathrm{Lu}^{1}$ \\ ${ }^{1}$ Jingzhou No 1 People's Hospital and First Affiliated Hospital of Yangtze University
}

November 13, 2021

\begin{abstract}
Lynch syndrome (LS) is an autosomal dominant hereditary disease, which is caused by mismatch repair (MMR) gene mutations in the germline of MLH1, MSH2, MSH6 and PMS2. LS patients can develop colorectal cancer, endometrial cancer (EC), etc, at the same or different time, so their prognosis are poor.

Lynch syndrome type II associated endometrial carcinoma: a case report and literature analysis

Hui-Juan $\mathrm{Lu}^{1^{*}}$,Cun-Jian Yi ${ }^{2, *}$, Jian Liu $1^{*}$, Yu-Qi Qiu ${ }^{2 *}$, Yong Chen ${ }^{2 *}$, Jin-Zhi Lu ${ }^{2 *}$

${ }^{1}$ The First Affiliated Hospital of Yangtze University, Jingzhou, Hubei, 434000, China

${ }^{2}$ The First People's Hospital of Jingzhou, Jingzhou, Hubei, 434000, China

* These authors contributed equally.

Correspondence Cun-Jian Yi, The First People's Hospital of Jingzhou, Jingzhou, Hubei, 434000, China. Email:Cunjianyi@163.com.Telephone number: 13339738010

Abstract: Lynch syndrome (LS) is an autosomal dominant hereditary disease, which is caused by mismatch
repair (MMR) gene mutations in the germline of MLH1, MSH2, MSH6 and PMS2. LS patients can develop
colorectal cancer, endometrial cancer (EC), etc, at the same or different time, so their prognosis are poor.
Currently, there is few researches about LS-EC in China, and no complete screening system, diagnosis
and treatment standards or guidelines have been formed. In this paper, we reviewed the clinical diagnosis
and treatment data of a patient with Lynch syndrome type II with endometrial cancer, combined with
the screening and management guidelines for Lynch syndrome and related literature, and summarized the
molecular pathogenesis, clinicopathological features, screening and management protocols of Lynch syndrome
related endometrial cancer. It is hoped that through the management measures of early screening, early
prevention, and early diagnosis and treatment, the incidence and mortality of endometrial cancer related to
Lynch syndrome can be reduced.
\end{abstract}

Keywords: Lynch syndrome; endometrial cancer; mismatch repair gene

Lynch syndrome (LS), formerly known as hereditary nonpolyposis colorectal cancer syndrome (HNPCC), is an autosomal dominant genetic disease caused by DNA mismatch repair gene (MMR) mutations, and has family clustering ${ }^{[1]}$. Abnormal or missing MMR function leads to phenotypic mutations and microsatellite instability (MSI), thus promoting the occurrence of cancer ${ }^{[2]}$. MMR gene mutations will greatly increase the probability of endometrial cancer, colorectal cancer, epithelial ovarian cancer, breast cancer, gastric cancer, bladder cancer, kidney cancer and gastric cancer among relatives ${ }^{[3]}$. Diagnosing LS patients and giving their relatives corresponding genetic counseling advice can significantly reduce the incidence of LS-related tumors. The clinical data of a patient with LS-EC admitted to the First People's Hospital of Jingzhou, 
the First Affiliated Hospital of Yangtze University is retrospectively analyzed, and combined with literature review, the report is as follows.

\section{Case presentation}

Here we report a woman who developed endometrial cancer at the age of 45 years, with no previous history of cancer. She had a family history of a first-degree relative who died of colorectal cancer at an unknown age. No other relevant family history was revealed. She is unmarried and childless, has asexual life history, G0, and underwent hysteroscopic surgery in this hospital for "endometrial polyps" in 2009. 2020/10/01 Pelvic ultrasound shows: Hypoechoic mass behind the cervix, subserosal fibroids? (about $78^{*} 58 \mathrm{~mm}$ ), hypoechoic mass of the posterior wall of the uterus (about 16*10mm), uterine fibroids. 2020/10/7: CA125: 41.90U/ml. 2020/10/10 MRI report shows: cervical-rectal space occupying lesions $\left(76^{*} 77^{*} 76 \mathrm{~mm}\right)$, the nature is to be determined; the endometrium is thickened, and there are strips of abnormal signal foci in the uterine cavity, which are separated from the myometrium Unclear, tumorous lesions need to be excluded; pelvic effusion ( Fig. 1). There were no contraindications in preoperative preparations, and laparoscopic exploration + pelvic adhesion lysis + transabdominal extensive hysterectomy + bilateral adnexectomy + pelvic lymphadenectomy were performed on 2020/10/13. During the operation, we could see: full uterus, fibroids with a diameter of $1 \mathrm{~cm}$ on the posterior wall, and the appearance of bilateral appendages was normal. A solid mass of $7 \mathrm{~cm}$ in diameter was seen between the posterior wall of the cervix and the rectum, which was densely adhered to the surrounding tissues. Separating the mass and adhesion of the rectum, we could see that the capsule was basically intact, part of it was densely adhered to the intestine, and the boundary with the rectum and surrounding tissues was clear, and there was no obvious invasion. The tumor was exposed in the capsule, and the tissue was fragile. Then the tumor was removed and sent for quick frozen section as malignant. Considering the possibility that the tumor originated from the reproductive organs, it was agreed to switch to laparotomy after explaining the condition to the family, and then extensive transabdominal hysterectomy + Bilateral appendectomy + pelvic lymphadenectomy were performed. We removed the mass in the rectum and vaginal diaphragm, incise the vaginal wall, and removed the tissue invaded by the tumor. The uterus and cervix were dissected with polypoid growths of $4 \mathrm{~cm}$ in diameter, and there were many small polypoid growths in the uterine cavity. Send a quick frozen section again and report as a cervical polyp, which does not exclude the possibility of endometrial cancer, which requires routine medical examination to confirm. The pelvic lymph node groups were sequentially removed, and the corresponding lymph vessels were ligated. Obviously enlarged lymph nodes were seen in the left and right internal iliac and the right common iliac.

Postoperative pathological reports: 1. Well-differentiated endometrioid adenocarcinoma, immunohistochemistry: $\mathrm{ER}(+) \mathrm{PR}(+) \mathrm{MLH1}(+) \mathrm{MSH} 2(-)$ MSH6(-)PMS2(+), MLH1 was expressed in cancer foci , PMS2 protein, localized in the cell nucleus, appeared light yellow, yellow or brown, while MSH2 and MSH6 protein were not expressed ( Fig. 2A-D). 2. (Pelvic floor, pelvic cavity) malignant tumors with partial necrosis, of which about $10 \%$ were moderately differentiated endometrioid adenocarcinoma, about $90 \%$ were considered to be mesenchymal malignant tumors, epithelioid leiomyosarcoma may be. 3. Cervical polyps with squamous metaplasia. 4. Uterine leiomyoma. The medical examination and consultation results of Wuhan Union Medical College Hospital showed that: well-differentiated endometrioid adenocarcinoma, pelvic floor, posterior cervical wall, and uterine serosa sarcomatoid carcinoma (carcinosarcoma) with large areas of necrosis, suggesting that the patient may have Lynch syndrome, suggest 17 \# And 29\# to organize MSH6 and MSH2 gene mutation detection. Combined with the patient's auxiliary examination and family history (mother died of "colon cancer"), a diagnosis of Lynch syndrome was considered, so gastrointestinal endoscopy and genetic testing were suggested to be made. 2020/11/12 gastroscope showed: chronic non-atrophic gastritis with erosion. 2020/11/12 colonoscopy showed: rectal space (extramural invasion?), the intestinal mucosa submitted for examination showed chronic inflammatory and acute active changes, with foci with rich cytoplasm, red stained, translucent cells, Ki67 Li approximately $20 \%$ was considered to be a rectal malignant tumor, and then based on the medical history, the possibility of sarcomatoid cancer is considered high.

Re-examination in one month after surgery: 2020/11/18 MRI showed: huge space-occupying lesions in the cervix-rectum space $(82 * 86 * 84 \mathrm{~mm})$, local gas accumulation in the lesion, and the lesion breaking through 
the rectum to grow into the rectal cavity, considering tumor recurrence . 2020/12/04 the genetic test report showed: Exon 5 of the MSH2 gene, a heterozygous nonsense mutation (exon5 c.C940T p.Q314X) was detected, which was a pathogenic mutation, suspected to be germline, combined with LS related, consider the MSI-H type. She was transferred to the oncology department for further diagnosis and treatment, namely chemotherapy and targeted combined immunotherapy: albumin paclitaxel + carrelizumab + bevacizumab. Re-examination after comprehensive treatment: 2021/7/22 MRI showed: postoperative changes in the uterus; vaginal stump space-occupying lesions $\left(18^{*} 22^{*} 17 \mathrm{~mm}\right)$, considering tumor recurrence, lesions involving the anterior wall of the rectum, vaginal stump-rectal fistula may be, The lesion was smaller than before. Tumor markers such as CA125 were normal after repeated examinations. Follow-up treatment is still in progress.

\section{Discussion}

\subsection{Molecular mechanism and classification of Lynch syndrome}

Lynch syndrome is the most common hereditary colorectal cancer syndrome, in which mutations in MLH1, MSH2, MSH6, and PMS2 account for approximately $37 \%, 41 \%, 13 \%$, and $9 \%$, respectively ${ }^{[4]}$. EPCAM is an upstream gene of MSH2. Its deletion causes hypermethylation of the MSH2 promoter, which in turn leads to its loss of expression ${ }^{[5]}$. MSH2 is not expressed in this patient's cancer foci. According to the MSI diagnostic criteria, the tumor is divided into 3 types: MSI-H, MSI-L and MSS ${ }^{[6]}$, the patient is MSI-H. According to different cancer sites, Lynch syndrome can be divided into two types: Type I (typical) is mainly characterized by: autosomal dominant genetic disease, which is mainly manifested as a large number of colorectal cancers at the same time or at different times, and some intestines Excessive appearance of external malignant tumors, early onset, often invaded the proximal colon, pathologically poorly differentiated, the survival rate of colon cancer is improved, and the subclinical adenocarcinoma accelerates malignant metastasis ${ }^{[7]}$. Type II (atypical): In addition to colorectal cancer, there are also extra-intestinal tumors, including endometrial cancer and other tumors ${ }^{[1]}$. The patient belongs to type II, atypical Lynch syndrome. He has no history of high risk factors for endometrial cancer and abnormal uterine bleeding, and has a history of dysmenorrhea. Preoperative color Doppler ultrasound revealed a huge subserosal uterine fibroids, and no obvious blood flow was seen. In addition, the patient is unmarried and childless, has no sex life history, has limited gynecological examinations, has not performed diagnostic curettage before surgery, and postoperative medical examinations suggest endometrial cancer. We should learn lessons from this case, and in the future, similar cases should undergo diagnostic curettage to confirm the diagnosis.

\subsection{Screening and diagnosis of endometrial cancer associated with Lynch syndrome}

According to reports, endometrial cancer is the first symptom of about $40 \%-60 \%$ of female LS patients. It is also the most common extraintestinal manifestation of type II LS, and is considered to be the "sentinel cancer" of LS ${ }^{[8]}$. This patient was also diagnosed with endometrial cancer first, and then rectal lesions were found. Combined with the results of genetic testing, LS was considered. Therefore, more and more scholars have begun to study Lynch syndrome-related endometrial cancer (LS-EC). LS-EC has the following characteristics: (1) The age of onset is 46 to 54 years old. (2) The pathological types are diverse and the degree of differentiation is low. (3) Most of the lesions are located in the lower part of the uterus. The patient was 45 years old. The pathological results showed adenocarcinoma and carcinosarcoma, and the lesion was located in the space between the posterior cervix and rectum, which basically met the above characteristics. In previous studies, there have been many reports about MLH1 and MSH2 mutations, and the patient's MSH2 gene was also detected with pathogenic mutations. Occasionally, MSH6 mutations can be detected in LS, while PMS2 mutations are relatively rare ${ }^{[9]}$. Regardless of the clinical diagnostic criteria used in patients with endometrial cancer, some LS-EC patients may be missed and misdiagnosed. Therefore, experts recommend MMR gene detection, MSI analysis, immunohistochemistry, BRAF detection, and MLH1 promoter methylation analysis for all women diagnosed with endometrial cancer to screen for Lynch syndrome ${ }^{[10-12]}$. In addition, tumor markers such as CA125 can also be used to screen for LS-EC [13]. The patient's blood CA125 was also elevated before the operation, and the CA125 was normal after the re-examination. Women with LS will have a great risk of developing EC as the first type of tumor, so it is 
necessary to determine whether EC is related to LS. The patient considered uterine fibroids before surgery, diagnosed endometrial cancer after surgery, combined with immunohistochemical results and family history, suspected LS, genetic testing confirmed LS, so it is recommended that the patient's family receive related genetic counseling. In the future, in the diagnosis and treatment of similar patients, it is necessary to improve the understanding of LS, improve gastrointestinal endoscopy and genetic testing and other examinations, so as to achieve early diagnosis and early treatment. The identification of EC as LS-related can also be used for screening, early detection and prevention of other tumors, such as colorectal cancer.

\subsection{Treatment and prevention of endometrial cancer associated with Lynch syndrome}

Although the pathogenesis and clinical manifestations of endometrial cancer associated with Lynch syndrome are different from sporadic endometrial cancer, the treatment is similar. The details are as follows: Progesterone is not applicable; Staged surgery, combined with radiotherapy and chemotherapy for stage II or higher; Lymph node dissection should be performed at the same time for cancer of the lower uterus; Radiotherapy may be considered when there are contraindications to surgery ${ }^{[14]}$; For MSI -H/MMR-D malignant tumor, immunotherapy has a long-lasting effect ${ }^{[15]}$. The results of Florine et al. suggest that MSI endometrial cancer is more suitable for anti-PD-1/PD-L1 immunotherapy ${ }^{[16]}$. In 2017, the FDA approved PD-1/PD-L1 inhibitors for the treatment of patients with solid tumors of MSI-H or d MMR. After the patients were treated with surgery, chemotherapy, and targeted combined immunotherapy, the effect was significant, and the lesions were significantly smaller than before ( Fig. 3), the current general situation is acceptable. Preventive treatment, including gynecological examination, transvaginal ultrasound, endometrial biopsy and colonoscopy, etc. Preventive surgical removal of the uterus and bilateral appendages can reduce the incidence of gynecological tumors ${ }^{[17-18]}$. Through these measures, early screening, early diagnosis, and early treatment of LS can be achieved, thereby reducing the morbidity and mortality of LS and its related tumors ${ }^{[19]}$.

The current domestic research on LS-EC is still in the initial stage. Further research on the pathogenesis and prevention methods of LS-EC will help improve the level of early screening, prevention, diagnosis and treatment of LS-EC, and reduce the risk of LS-EC. Morbidity and mortality can improve the survival time of patients.

\section{Declarations}

\section{1)Consent to publication}

We declare that all authors agreed to publish the manuscript at this journal based on the signed Copyright Transfer Agreement, and followed publication ethics.

\section{2)Ethical approval and consent to participants}

Not applicable.

\section{3)Disclosure of conflict of interests}

We declare that no conflict of interest exists.

\section{4)Funding}

This work was supported by Hubei Provincial Health and Family Planning

Commission Medical College Special Project (Key Project) (WJ2018H175).

\section{5)Availability of data and material}

We declare that the data supporting the results reported in the article are available in

the published article.

\section{6)Authors' Contributions}


Authors contributed to this paper with the design (CJY), literature search

(HJL,JL,YQQ), drafting (HJL), revision (CJY and JZL), editing (HJL and CJY) and

final approval (CJY).

\section{7)Acknowledgements}

None

\section{8)Authors' biography}

None

\section{References:}

[1] Guo Yanping, Yang Chunbao, Li Yang et al. A case of Lynch syndrome and literature review [J]. Journal of Chinese Academy of Medical Sciences. 2018. 40(03): p. 432-435.

[2] Guo Chao, Liu Aijun. Progress in the pathology of endometrial cancer associated with Lynch syndrome [J]. Journal of Chinese People's Liberation Army Medical College. 2015. 36(06): p. 640-643.

[3] Lynch, H.T., Snyder CL, Shaw TG., et al., Milestones of Lynch syndrome: 1895-2015 [J]. Nat Rev Cancer, 2015. 15(3): p. 181-94.

[4] Moreira, L., Balaguer, F., Lindor, N., et al., Identification of Lynch syndrome among patients with colorectal cancer [J]. Jama, 2012. 308(15): p. 1555-65.

[5] Zhang Qun, Wang Ling, Han Liying, et al. Research progress in endometrial cancer associated with Lynch syndrome [J]. International Journal of Obstetrics and Gynecology. 2019. 46(05): p. 564-567.

[6] Rodriguez-Bigas, MA, Boland CR, Hamilton SR, et al., A National Cancer Institute Workshop on Hereditary Nonpolyposis Colorectal Cancer Syndrome: meeting highlights and Bethesda guidelines [J]. J Natl Cancer Inst, 1997. 89(23): p. 1758-62.

[7] Wang Tiantian, Zhang Rong. Lynch syndrome and gynecological tumors [J]. International Journal of Obstetrics and Gynecology. 2016. 43(05): p. 497-501.

[8] Lu, KH, Kohlmann W, Watson P, et al., Gynecologic cancer as a "sentinel cancer" for women with hereditary nonpolyposis colorectal cancer syndrome [J]. Obstet Gynecol, 2005. 105(3): p. 569 -74.

[9] Cui, MH, Zhang XW, Yu, T., et al., PMS2 germline mutation c.1577delA (p.Asp526Alafs*69)-induced Lynch syndrome-associated endometrial cancer: A case report [J]. Medicine ( Baltimore), 2019. 98(51): p. e18279.

[10] Kahn, RM, Gordhandas, S., Maddy, BP, et al., Universal endometrial cancer tumor typing: How much has immunohistochemistry, microsatellite instability, and MLH1 methylation improved the diagnosis of Lynch syndrome across the population [J]. Cancer, 2019. 125(18): p. 3172-3183.

[11] Hendriks, YM, Jong, AE, Morreau, H., et al., Diagnostic approach and management of Lynch syndrome (hereditary nonpolyposis colorectal carcinoma): a guide for clinicians [J]. CA Cancer J Clin, 2006. 56 (4): p. 213-25.

[12] Giardiello, FM, Allen, JI, Axilbund, JE, et al., Guidelines on genetic evaluation and management of Lynch syndrome: a consensus statement by the US Multi-Society Task Force on Colorectal Cancer [J]. Dis Colon Rectum , 2014. 57(8): p. 1025-48.

[13] Wang Fang, Yu Qianqian, Li Dan, et al. One case report of endometrial cancer related to Lynch syndrome [J]. Chinese Journal of Laboratory Diagnosis. 2019. 23(10): p. 1785-1786.

[14] Yu Yue, Yuan Jirong. New progress in endometrial cancer associated with Lynch syndrome [J]. Chinese Journal of Eugenics and Genetics. 2016. 24(10): p. 134-136. 
[15] Biller, L.H., Syngal, S., and Yurgelun, M.B., et al., Recent advances in Lynch syndrome [J]. Fam Cancer, 2019. 18(2): p. 211-219.

[16] Florine, A., Eggink, FA, Van Gool, IC, et al., Immunological profiling of molecularlyclassified highrisk endometrial cancers identifies POLE-mutant and microsatellite unstable carcinomas as candidates for checkpoint inhibition [J]. Oncoimmunology, 2017 , 6(2): e1264565.

[17] Rabban, JT, Calkins, SM, Karnezis, AN, et al. Association of tumor morphology with mismatch-repair protein status in older endometrial cancer patients: implications for universal versus selective screening strategies for Lynch syndrome [J]. Am J Surg Pathol, 2014, 38(6): 793-800.

[18] Liu Dajiang, Du Wenjing, Yang Yongxiu. New advances in endometrial cancer associated with Lynch syndrome [J]. International Journal of Obstetrics and Gynecology. 2016. 43(01): p. 67-69.

[19] Half, E.E. and R.S. Bresalier, Clinical management of hereditary colorectal cancer syndromes [J]. Curr Opin Gastroenterol, 2004. 20(1): p. 32-42.

[20] Hagen, CE, Lefferts, J., Hornick, JL, et al., "Null pattern" of immunoreactivity in a Lynch syndromeassociated colon cancer due to germline MSH2 mutation and somatic MLH1 hypermethylation [J]. Am J Surg Pathol, 2011. 35(12): p. 1902-5.

[21] Raymond, VM, Morris, AM, Hafez, KS, et al., MLH1 promotor hypermethylation does not rule out a diagnosis of Lynch syndrome: a case report [J]. Fam Cancer, 2015. 14(1): p . 77-80.

[22] Rahner, N., Friedrichs, N., Steinke, V., et al., Coexisting somatic promoter hypermethylation and pathogenic MLH1 germline mutation in Lynch syndrome [J]. J Pathol, 2008. 214(1): p. 10-6.

[23] Yokoyama, T., Friedrichs, N., Steinke, V., et al., Lynch syndrome-associated endometrial carcinoma with MLH1 germline mutation and MLH1 promoter hypermethylation: a case report and literature review [J]. BMC Cancer, 2018. 18(1): p. 576.

[24] Seiden, MV, Patel, D., O'Neill, MJ, et al., Case records of the Massachusetts General Hospital. Case 13-2007. A 46-year-old woman with gynecologic and intestinal cancers [J] . N Engl J Med, 2007. 356(17): p. $1760-9$.

[25] Lee, HJ, Lee, MH, Choi, MC, et al., Case Report of Menopausal Woman Diagnosed with Endometrial Cancer after Colon Cancer with Germline Mutation in MSH6 in Korea [J]. J Menopausal Med, 2017. 23( 1): p. 69-73.

[26] Chapel, DB, Lengyel, E., Ritterhouse, LL, et al., Interpretation of Mismatch Repair Protein Immunohistochemistry in Endometrial Carcinoma Should Consider Both Lynch Syndrome Screening and Immunotherapy Susceptibility: An Illustrative Case Report [J]. Int J Gynecol Pathol, 2020. 39(3): p. 233-237.

[27] Nakamura, K., Nakayama, K., Minamoto, T., et al., Lynch Syndrome-Related Clear Cell Carcinoma of the Cervix: A Case Report [J]. Int J Mol Sci, 2018. 19(4). 

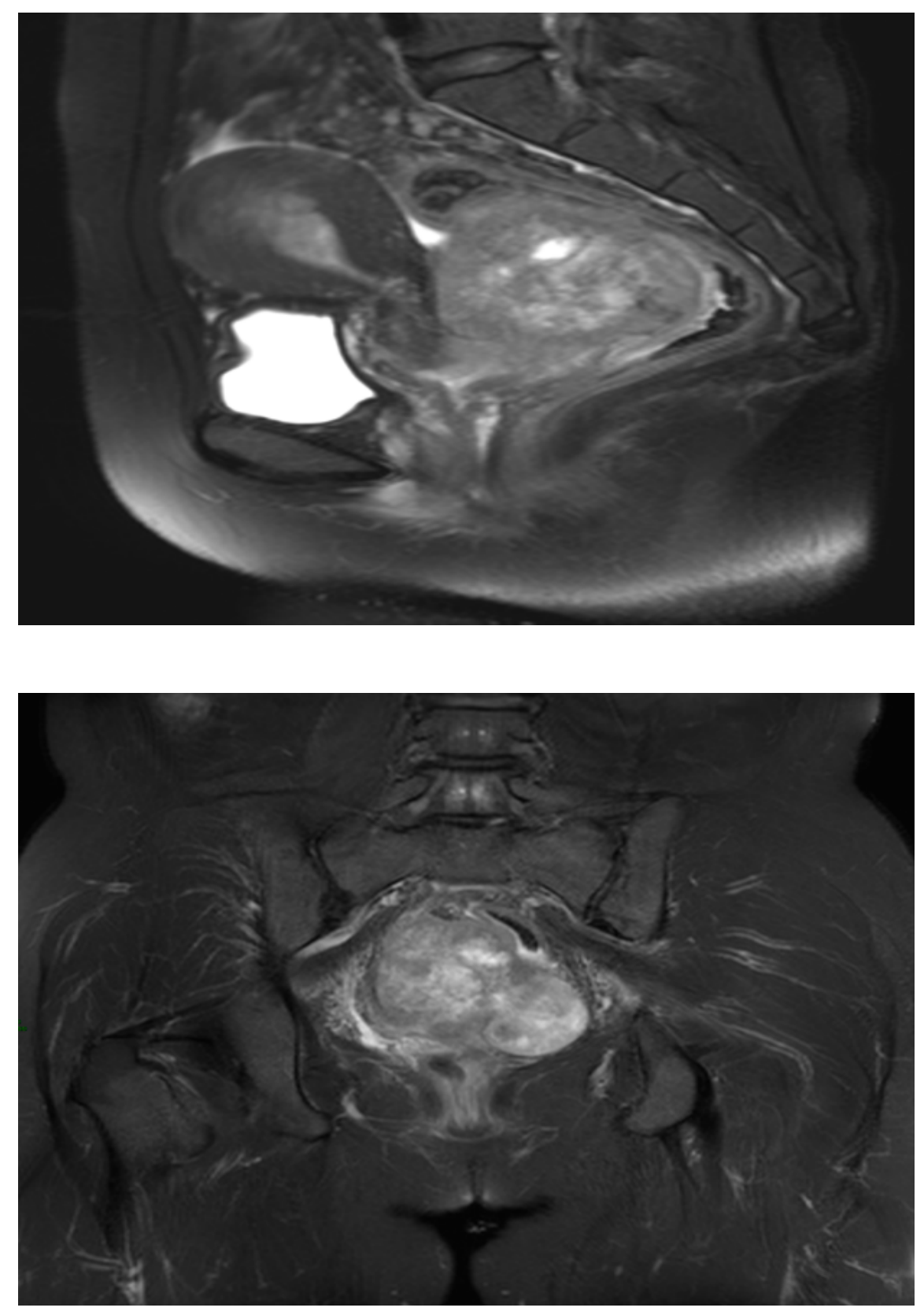

Left image: pelvic MRI sagittal plane; right image: pelvic MRI coronal plane

Fig. 1 : MRI of the pelvis 

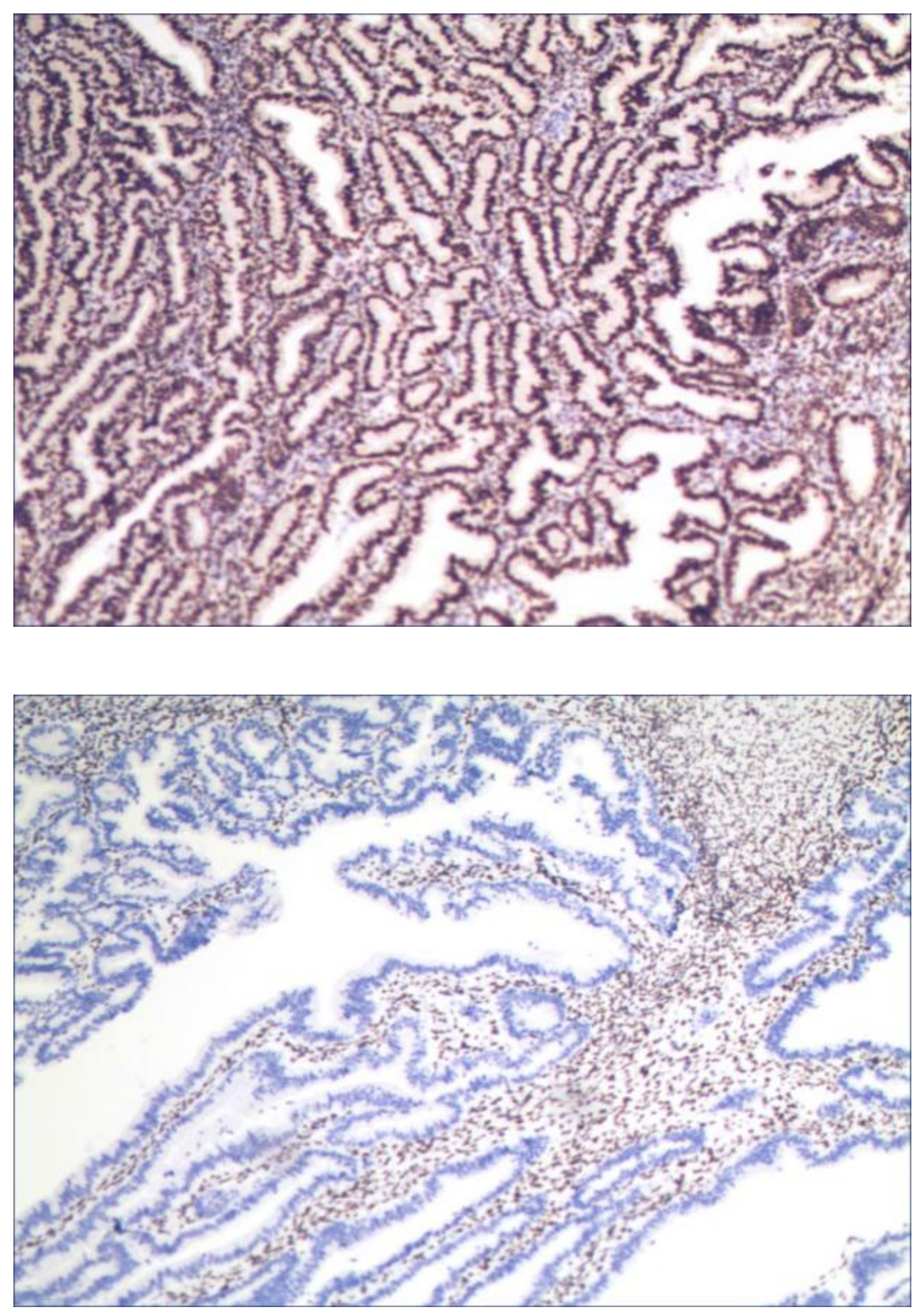

A.MLH1 B.MSH2 

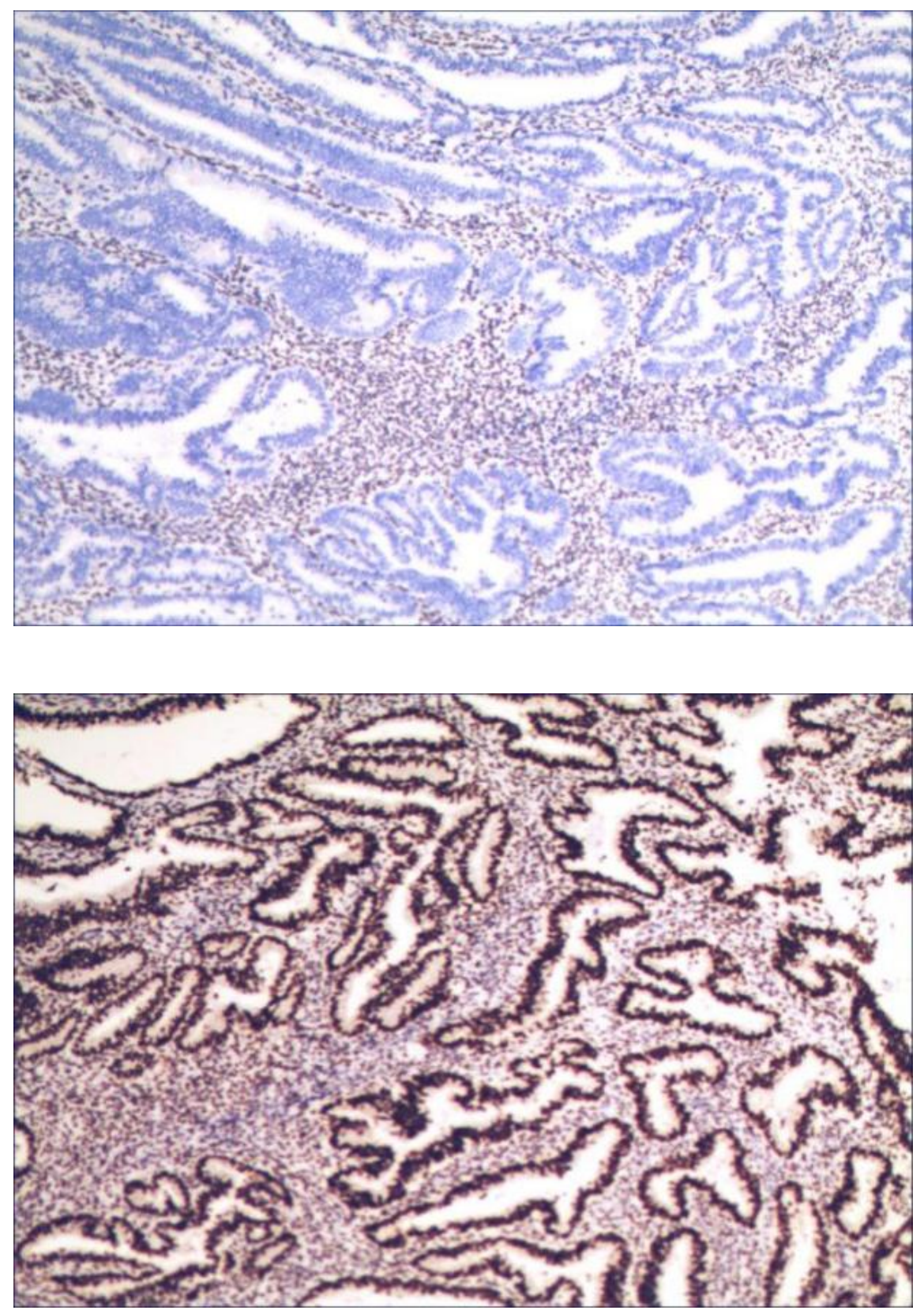

\section{MSH6 D.PMS2}
A. MLH1 protein stains positive in cancer cells;
B. MSH2 protein stains negative in cancer cells;
C. MSH6 protein is negative in cancer cells;
D. PMS2 protein stains positive in cancer cell

Fig. 2 : The results of immunohistochemical staining of MMR protein in surgical specimens of endometrial cancer $(\times 100)$; 

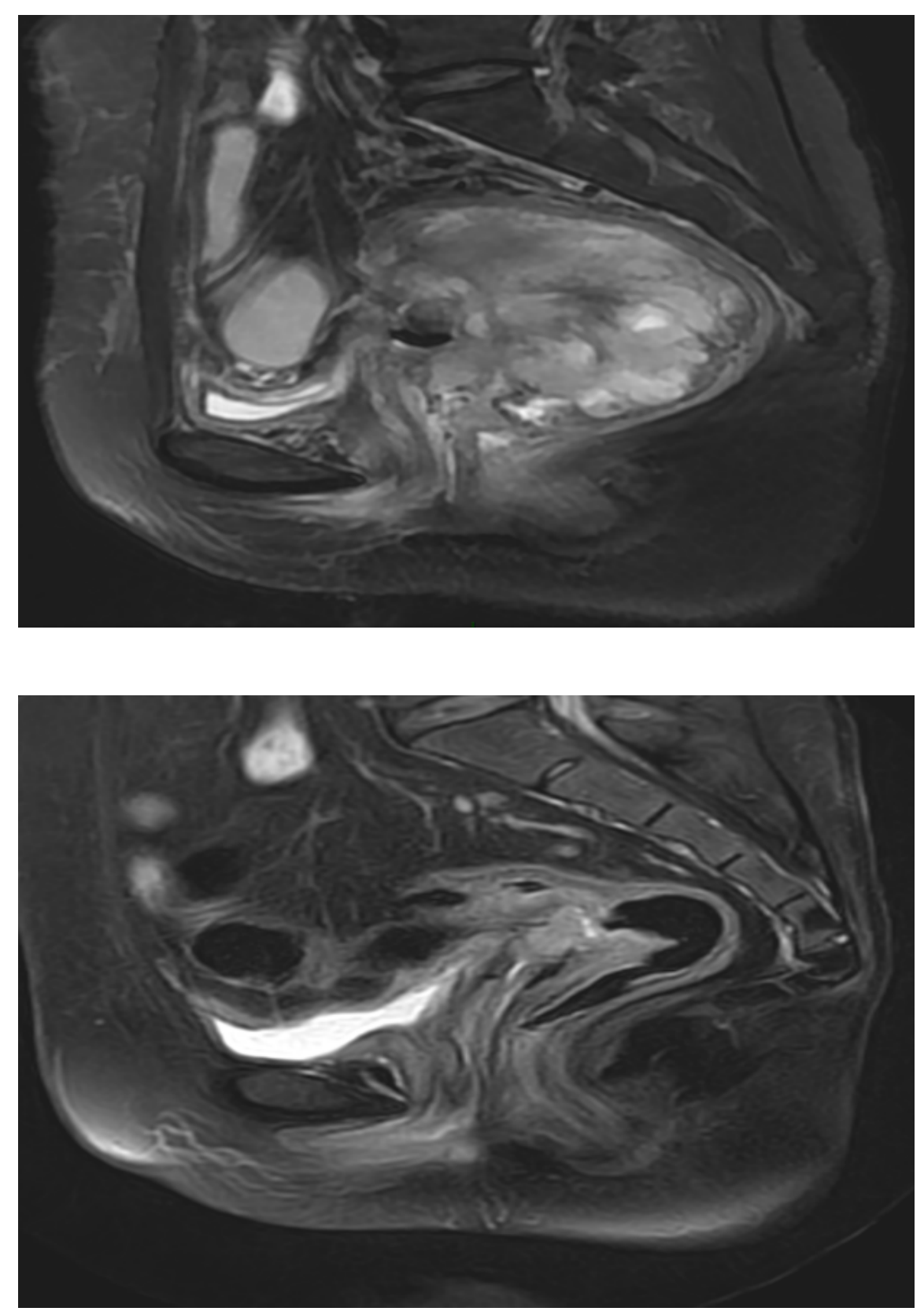

Left image: Vaginal stump lesion; Right image: Anterior rectal wall lesion

Fig. 3 : Sagittal view of pelvic MRI 


\section{Table 1}

Summary of Lynch syndrome related cases:

MLH1

hypermethylation and germline mutations of mismatch repair genes coexist

Case Author Age/Year Tumor Type

1 Hagen et al. ${ }^{[20]}$ 71 CRC

2 Raymond et al. ${ }^{[21]} 75$

CRC、OvCa

3 Rahner et al. ${ }^{[22]}$

N/A CRC

4 Yokoyama et al. $^{[23]} 49 \mathrm{EC}$
Table 1

Summary of Lynch syndrome related cases: MLH1 hypermethylation and germline mutations of mismatch repair genes coexist IHC MLH1 MSH2 MSH6 PMS2 MSI

- - - N/A

- + - - MSI-H

-++ - MSI-H

$-++-\mathrm{N} / \mathrm{A}$

5 Seiden et al. ${ }^{[24]} 46+--+$ N/A CRC、EC、OvCa
Table 1

Summary of Lynch syndrome related cases: MLH1 hypermethylation and germline mutations of mismatch repair genes coexist germline mutation

MSH2

MSH6

MLH1

MLH1

MSH2
Table 1

Summary of Lynch syndrome related cases: MLH1 hypermethylation and germline mutations of mismatch repair genes coexist BRAF mutation

wild type

wild type

$\mathrm{N} / \mathrm{A}$

$\mathrm{N} / \mathrm{A}$

$\mathrm{N} / \mathrm{A}$

$+\mathrm{N} / \mathrm{A} \mathrm{N} / \mathrm{A}$

$\mathrm{N} / \mathrm{A}$

$+--+\mathrm{N} / \mathrm{A}$

+--+ MSI-H
MSH6

$\mathrm{N} / \mathrm{A}$

MSH2/MSH6

$\mathrm{N} / \mathrm{A}$

$\mathrm{N} / \mathrm{A}$

$\mathrm{N} / \mathrm{A}$
Table 1

Summary of Lynch syndrome related cases:

MLH1

hypermethylation and germline mutations of mismatch repair genes coexist

Family history

Mother:

OvCa;son: CRC;Daughter: breast, CRC

Father - brother: CRC

$\mathrm{N} / \mathrm{A}$

First-degree relative: CRC; Second-degree relative:gastric cancer

Mother: EC 、 basal cell carcinoma; grandfather: CRC;grandmother: breast cancer

Sister:

EC;brother:CRC $\mathrm{N} / \mathrm{A}$

Not obvious al. ${ }^{[27]} 42$ cervical cancer

IHC: immunohistochemistry colorectal cancer: CRC ovarian cancer: OvCa N/A: not available EC: endometrial cancer 